\title{
LEITUNGSPRAXIS
}

\section{Gesund führen}

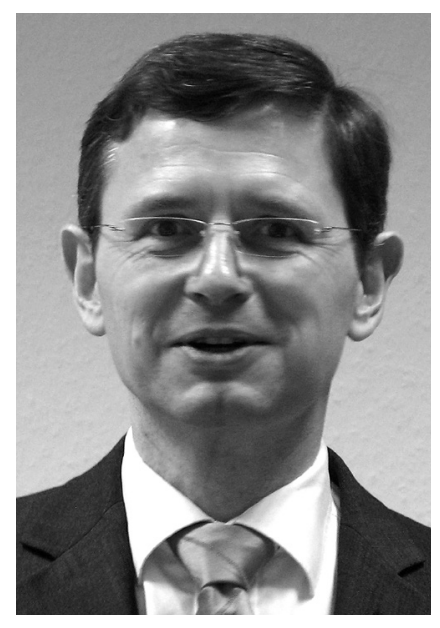

VON FRANK STÖPEL

Dr. Frank Stöpel, Diplom-Pädagoge, promovierte in Psychologie über den Zusammenhang von Arbeit und Gesundheit. Seit über 15 Jahren ist er Personalentwickler mit den Schwerpunkten Führung, Gesundheit und betriebliches Eingliederungsmanagement. www.dr-stoepel.de

\author{
Die arbeitswissenschaftliche Forschung hat \\ zahlreiche Faktoren identifiziert, die krank machen \\ oder die gesund erhalten. Für den Führungsalltag \\ sind all diese Ergebnisse oft zu umfassend und \\ eher verwirrend als klärend. Als pragmatische \\ Orientierung kann die Theorie des Kohärenzgefühls \\ dienen, die drei bedeutsame Faktoren ermittelt hat: \\ Verstehbarkeit, Handhabbarkeit, Sinnhaftigkeit.
}

Ein schlechter Chef kann einen Mitarbeiter schneller und umfassender krankmachen, als ein ergonomisch schlecht gestalteter Arbeitsplatz dazu in der Lage wäre. Dies zeigt nicht nur die Erfahrung, sondern wird auch durch die wissenschaftliche Forschung bestätigt. $\mathrm{Zu}$ erklären ist dies dadurch, dass Führungskräfte wesentlich die Art und Inhalte der Kommunikation bestimmen, die Arbeit organisieren (auch eine fehlende Organisation ist eine spezifische Organisation der Arbeit), Feedback geben und auch die Atmosphäre am Arbeitsplatz prägen. Dadurch wird auch beeinflusst, wie die Kollegen untereinander umgehen, wie mit Themen wie Lob und Kritik umgegangen wird, wie Konflikte gelöst werden, wie der Umgang mit Andersartigkeit ist.

Die Frage stellt sich, wie diese "weichen « Faktoren sich auf die Gesundheit der Mitarbeiter auswirkt. Man muss sich von einer Vorstellung verabschieden, dass man entweder gesund oder krank ist. Jeder Mensch ist mehr oder weniger gesund und krank. Gesundheit und Krankheit sind nur die Extrempunkte eines Kontinuums, auf dem wir uns fortwährend bewegen. Es gibt Belastungsfaktoren, die uns tendenziell in Richtung Krankheit bewegen. Hierzu zählen physikalische Belastungsfaktoren (Lärm, Hitze, Staub usw.), welche in den letzten Jahren kontinuierlich redu- ziert worden sind. Was übrig geblieben ist, sind die psychischen Belastungen, welche in der Norm EN ISO 10075 als »die Gesamtheit aller erfassbaren Einflüsse, die von außen auf den Menschen zukommen und psychisch auf ihn einwirken « definiert worden sind. Gerade bei der Erfassung und Reduzierung der psychischen Belastungen haben viele Unternehmen und Organisationen noch erheblichen Handlungsbedarf. Die Belastungen beschreiben die von außen auf den Menschen einwirkenden Faktoren, seine spezifische Reaktion wird als Beanspruchung beschrieben. Beanspruchungsfolgen sind Ermüdung, Stress und Krankheit. Vereinfacht kann man sagen: je höher die Beanspruchung, desto höher ist das Risiko für eine Erkrankung.

Der israelische Medizinsoziologie Aaron Antonovsky hat sich seit den 1970er Jahren mit der Frage beschäftigt, warum Menschen trotz Belastungen gesund bleiben. Die Antwort auf diese Frage fand er in einer überdauernden Einstellung: Das Leben ist danach charakterisiert durch ein Gefühl der Verstehbarkeit, der Handhabbarkeit und der Sinnhaftigkeit. Diese Orientierung nannte Antonovsky das Kohärenzgefühl. Zahlreiche internationale Untersuchungen haben inzwischen gezeigt, dass Menschen mit einem ausgeprägten Kohärenzgefühl erfolgreicher mit schwierigen Situationen umgehen können. 


\section{Verständlichkeit}

"Ich weiß gar nicht, warum sich meine Mitarbeiter beschweren, dass sie zu wenig Informationen bekommen. Alles, was ich an E-Mails und Umläufen bekomme, leite ich an sie weiter", meint eine Führungskraft. Doch die Herausforderung besteht nicht darin, Informationen zu bekommen, sondern die wirklich relevanten zu erkennen. Viele Beschäftigte leiden unter der Informationsflut und es fällt schwer, mit der Masse der täglichen Nachrichten umzugehen. Eine Aufgabe bei "gesund führen" ist entsprechend, die Informationsflut für die Mitarbeiter durch Auswahl, Strukturierung und Kommentierung zu begrenzen.

Ein weiterer Aspekt für das Gefühl der Verständlichkeit sind klare Abläufe und Strukturen: Wer macht was wann und wie. Und vor allem: Kann sich der Mitarbeiter darauf verlassen, dass diese Strukturen Bestand haben, oder werden spontan Führungsebenen bei Anweisungen übersprungen. Die Kunst besteht darin, bei aller Flexibilität klare und verlässliche Strukturen zu etablieren.

\section{Handhabbarkeit}

Gerade in der aktuellen Diskussion um Burn-out wird nochmals deutlich, wie wichtig es ist, Überforderung zu vermeiden. Aus der Diskussion um Zielvereinbarungen ist bekannt, dass Ziele, welche eine subjektiv 50-prozentige Erfolgsaussicht haben, zu maximaler Anstrengung führen. Die erfolgreiche Bewältigung schwieriger Aufgaben erhöht auch das Gefühl der Selbstwirksamkeit.

In der Praxis finden die Mitarbeiter aber nicht immer Kriterien vor, an denen sie den Erfolg ihrer Anstrengung messen können oder eine Rückmeldung dazu erhalten. Oft finden sich auch Führungskräfte, welche Erfolge in ihrem Verantwortungsbereich öffentlich sich selber zuschreiben, Gründe für die Misserfolge aber bei den Mitarbeitern verorten. Auch der berüchtigte »Dienst nach Vorschrift " ist in diesem Zusammenhang zu sehen: Wenn ein Mitarbeiter das volle Risiko bei Eigeninitiative trägt, dann ist es doch nur vernünftig, dass er sich absichert und sich nur im Rahmen der Vorschriften bewegt. Man kann es auch als gelernte Hilflosigkeit beschreiben, wenn Mitarbeiter aufhören sich zu engagieren, weil sie wiederholt erleben mussten, dass sie doch nichts bewegen konnten.

Es gilt, sowohl Über- als auch Unterforderung zu vermeiden. Menschen sind unterschiedlich und verändern sich fortlaufend. Daher ist es für Führungskräfte auch nicht immer leicht, was für einen spezifischen Mitarbeiter aktuell eine Über- oder Unterforderung darstellt. Oft hilft nur eins: Nachfragen.

Die psychologische und pädagogische Forschungsliteratur ist voll mit Hinweisen darauf, wie wichtig Fehler für die Kompetenzentwicklung sind. Auch das moderne Qualitätsmanagement will die Fehler erfassen, damit daraus gelernt werden kann und zukünftige, womöglich schwerere Fehler vermieden werden können. Eine positive Fehlerkultur ist nicht nur für die Qualitätssicherung notwendig, sondern auch damit die Mitarbeiter angstfrei ihre Aufgaben angehen und sich weiter entwickeln können.

\section{Sinnhaftigkeit}

Ein Gefühl der Sinnhaftigkeit und Bedeutsamkeit hat im Zusammenhang der Arbeit zwei Aspekte: »Ist meine Arbeit wichtig? « und »Bin ich als Mensch wichtig?«. Auf die erste Frage vermag eine vollständige Handlung eine gute Antwort zu geben, da hier die Aufgabe auch in einen größeren Zusammenhang gestellt wird. Wenn Mitarbeiter keine Antwort finden auf die Frage, wofür sie sich anstrengen sollen, dann bleibt nur noch die Antwort: "Damit ich meine Brötchen bezahlen kann « und die einzige reizvolle Perspektive wird die Rente.

Mehrmals wurde mir berichtet, dass Führungskräfte den gleichen Arbeitsauftrag an zwei Mitarbeiter gaben, ohne dass diese von dem gleichen Auftrag an den anderen informiert wurden. Genommen wurde dann das Arbeitsergebnis, das der Führungskraft besser gefiel. Der andere Mitarbeiter hat dann halt für den Papierkorb gearbeitet. In der Praxis kommt es leider viel zu oft vor, das Arbeiten für den Papierkorb gemacht werden, auch ohne, dass solche Situationen mutwillig konstruiert werden. Hier ist es eine Herausforderung für die Führungskräfte, den Mitarbeitern zu vermitteln, wo der Sinn solcher Aufgaben liegt und warum es sich lohnt, sich weiter zu engagieren.

Jeder Mensch will wichtig und respektiert sein. Die Berichte von Mobbing zeigen, wie krank das Gegenteil machen kann. Wenn nicht gemobbt wird, beutet das noch lange nicht, dass Respekt und Wertschätzung vorliegen. Wie oft hören Vorgesetzte aufmerksam zu, wenn Mitarbeiter von ihren (beruflichen) $\mathrm{He}$ rausforderungen, Zielen und Träumen berichten? Wird der Reinigungskraft der verdiente Respekt gezollt für ihre schwere Arbeit, die schlecht bezahlt und niedrig angesehen wird?

Es gibt Führungskräfte, die sehr viel loben. Dann wissen die Mitarbeiter, dass der Chef entweder wieder auf einem Seminar war oder vom Mitarbeiter etwas will. Auch Führungskräfte, die jeden und alles loben, können nicht das Gefühl der Wertschätzung vermitteln. Respekt und Wertschätzung vermitteln sich nicht als manipulatives Mittel oder nach dem Gießkannenprinzip. Mitarbeiter bekommen es sehr gut mit, ob ein Lob ehrlich gemeint und berechtigt ist und auf einer Grundhaltung von Wertschätzung und Respekt dem Mitarbeiter gegenüber beruht.

\section{Fazit}

"Eine Führungskraft wird daran gemessen, was sie bewirkt und nicht daran, was sie macht", lautet ein Leitspruch. Gerade moderne Organisationen haben im Rahmen ihres betrieblichen Qualitätsmanagements auch Gesundheitsaspekte als Zielindikatoren definiert. Unabhängig davon ist es für jede Führungskraft attraktiv, gesund zu führen, weil sie hierdurch ihre Ziele einfacher erreichen kann. "Jede Führungskraft hat die Mitarbeiter, welche sie verdient", lautet ein weiterer Leitspruch, der in dieser Radikalität nicht zu halten ist. Es gibt auch Situationen und Mitarbeiter, die machen es Führungskräften nicht leicht, gesund zu führen. Trotzdem muss sich jeder Vorgesetzte bei demotivierten und kranken Mitarbeitern fragen, was er dazu beigetragen haben könnte.

Nicht krankmachende Führung versucht die belastenden Faktoren zu minimieren, gesunde Führung sucht, welche Faktoren die Ressourcen stärken. Damit Führungskräfte in ihrem Verantwortungsbereich eine Führungs- und Arbeitskultur entwickeln können, welche die Faktoren des Kohärenzgefühls stärken, benötigen auch sie Unterstützung. Mit einem Beschluss der Geschäftsführung " Ab heute wird gesund geführt « ist keinem geholfen. 